\title{
El territorio como espacio contradictorio: promesas y conflictos en torno a la actividad extractiva en Ecuador, Colombia, Perú y Chile
}

Juan Fernández-Labbé. Universidad Católica Silva Henríquez, Santiago, Chile.

RESUMEN | Los conflictos por actividades extractivas han aumentado en la última década y constituyen un desafío para los países en su deseo de avanzar en crecimiento económico y bienestar socioambiental. Se analiza aquí los casos de Ecuador, Colombia, Perú y Chile, poniendo en el centro el territorio como espacio social. Se argumenta que los conflictos representan un cuestionamiento al rol de los agentes, tanto privados como públicos, y empujan hacia la puesta en marcha de procesos de cambio institucional que suponen: a) fomentar y refinar los instrumentos jurídicos de captura de excedentes derivados de la actividad extractiva, para dirigirlos a inversión en otras áreas de desarrollo; b) desarrollar instrumentos de planificación y ordenamiento territorial; c) definir procesos de consulta previa; d) avanzar en descentralización y fortalecimiento de los gobiernos subnacionales; y e) fortalecer la capacidad de articulación de los actores involucrados para arribar a arreglos institucionales de crecimiento económico y desarrollo inclusivo.

PAlabras Clave | conflicto social, desarrollo territorial, transformaciones socioterritoriales.

ABSTRACT | The conflicts over extractive activities have grown in the last decade and represent a challenge for the countries, in their desire to advance in economic growth and socio-environmental welfare. The cases of Ecuador, Colombia, Peru and Chile are analyzed, placing the territory as a social space in the center. It is argued that conflicts represent a questioning of the role of both private and public agents, and press towards the implementation of processes of institutional change, which involve: a) promoting and refining legal instruments to capture surplus derived from the extractive activity, to direct them to investment in other areas of development; b) develop planning and territorial ordering instruments; c) define prior consultation processes; d) advance in decentralization and strengthening of subnational governments; and e) strengthen the articulation capacity of the actors involved to arrive at institutional arrangements for economic growth and inclusive development.

KEYWORDS | social conflict, territorial development, socio-territorial transformations.

Recibido el 22 de marzo de 2018, aprobado el 19 de septiembre de 2018.

E-mail: juanfernandezy@gmail.com 


\section{Introducción}

Los países de América Latina han registrado en las últimas décadas un importante crecimiento económico; sin embargo, pese a mejorar en algunos indicadores sociales, las brechas de desigualdad sociales y territoriales siguen estando presentes (Rimisp, Centro Latinoamericano para el Desarrollo Rural, 2012a; 2014; 2016; 2018). Ese crecimiento económico se ha basado esencialmente en un modelo de economía extractiva que somete a los territorios a fuertes impactos sociales y ambientales, configurando como consecuencia una alta conflictividad: más de 800 conflictos en América Latina (Atlas Global de Justicia Ambiental, 2018) y más de 200 en torno a la actividad minera solo en los cuatro países estudiados en el presente artículo: Ecuador, Colombia, Perú y Chile (Observatorio de Conflictos Mineros de América Latina [OCMAL], 2018). Lo anterior dificulta las opciones de desarrollo de los países y, a nivel territorial, opera "sacrificando" zonas y espacios sociales y culturales en pos de un prometido beneficio nacional, que se distribuye desigualmente. ${ }^{1}$

El objetivo del artículo es analizar los instrumentos institucionales utilizados por los países en torno a la actividad extractiva-minera a fin de prevenir y gestionar los conflictos derivados de ella, incorporando la mirada territorial. La metodología aplicada es la de estudio de casos a partir de una revisión documental que considera los cuatro países de la región andina señalados, los cuales presentan un diverso número de conflictos en torno a la minería. A partir de los resultados obtenidos, se proponen algunos ámbitos de acción para avanzar en arreglos institucionales encaminados hacia el desarrollo inclusivo y la equidad territorial.

El documento se organiza en cuatro apartados. El primero presenta una revisión conceptual y de antecedentes sobre territorio, extractivismo y conflictos socioterritoriales. El segundo describe el método de investigación y las fuentes de información. El tercero avanza en un análisis de los instrumentos públicos desplegados por los cuatro países en torno a la actividad extractiva-minera en el contexto descrito. Finalmente, se cierra con una reflexión en torno a cómo avanzar hacia una gobernanza territorial para el desarrollo inclusivo, en contextos de conflictividad.

\section{Antecedentes y marco conceptual}

\section{El territorio como espacio social y político}

El territorio, más allá de lo físico, es un "conjunto de relaciones sociales que dan origen y a la vez expresan una identidad y un sentido de propósito compartidos" (Schejtman \& Berdegué, 2004, p. 5). La noción de territorio alude a un espacio social que es apropiado y significado por sujetos y grupos que se afirman por medio de él, y en el cual hay relaciones sociales en tensión y diversas territorialidades (Porto-Gonçalves, 2009). El territorio pone límites y en él se da una relación social de poder que lo produce y lo mantiene. Esto determina la existencia de

1 Aparejado al marco general de conflictividad territorial, en 2017 se registraron 212 muertes en América Latina (Front Life Defenders [FLD], 2018, p. 12). FDL (2019) indica que líderes defensores de los derechos humanos y ambientales han sido víctimas de agresiones, campañas de difamación, amenazas a la seguridad digital, hostigamiento judicial y ataques de género. 
confrontaciones (Fernandes, 2005), en las cuales entran en juego estrategias e intereses de distintos actores donde interactúan lo económico, lo político, el desarrollo y el medioambiente (Bebbington, 2011a).

En los territorios circulan historias, significados, discursos, personas, bienes y servicios. Son espacios sociales de oportunidades y también de limitaciones. En América Latina, los territorios encarnan tanto las bondades del bienestar como los sacrificios de la pobreza, pues una parte significativa de los territorios está sumida en trampas de pobreza y desigualdad (Bebbington, Escobal, Soloaga \& Tomaselli, 2016). El destino de las personas depende de manera importante del territorio en que nacen y viven, donde interactúan estructuras y arreglos institucionales, actores internos y externos, mercados e intercambios económicos, que definen en la práctica sus características y potencialidades de desarrollo (Berdegué, Bebbington \& Escobal, 2015).

Economía extractiva: de dulce y de agraz para el desarrollo

La economía extractiva ha caracterizado la historia económica y el desarrollo de América Latina. ${ }^{2}$ Desde la conquista y la colonización, la región participó del proceso de estructuración del sistema capitalista mundial bajo la modalidad de acumulación extractivista, en aquel tiempo de la mano de la plata y el oro (Acosta, 2009; Seoane, 2012) y posteriormente sobre la base de un conjunto de minerales, hidrocarburos y materias primas forestales y pesqueras. Asociado a un crecimiento económico significativo -base de su discurso legitimador-, el extractivismo presenta dos ámbitos negativos. En primer lugar, configuraría una cultura económica rentista, que al depender de la explotación de la naturaleza ahoga la capacidad de innovación y riesgo empresarial y fomenta arreglos institucionales clientelares, dificultando que los países abundantes en recursos naturales logren ser desarrollados ${ }^{3}$ -lo que se ha denominado "la maldición de la abundancia"- (Karl, 1997). En segundo lugar, el crecimiento acelerado de las actividades extractivas acarrea fuertes impactos económicos, sociales y ambientales, que generan malestar y conflictos con las comunidades (Acosta, 2009; Bebbington, 2009; Gudynas, 2009). Lo anterior se ha visto potenciado tras las reformas neoliberales que han desregulado la economía (Bury, 2011a).

Analizando la participación de la producción de América Latina en el mercado mundial, se observa una alta concentración en recursos naturales y en manufacturas derivadas de ellos. El ańo 2010, el 57\% del valor de las exportaciones de la región correspondía a productos primarios y manufacturas basadas en recursos naturales, llegando a un 75\% en los países de Sudamérica (Comisión Económica para América Latina y el Caribe [Cepal], 2012).

El principal producto exportado por los países estudiados en el período 20002010 no solo no ha cambiado, sino que ha aumentado en valor dentro del conjunto

2 Entendiendo por economía extractiva las actividades económicas que remueven grandes volúmenes de recursos naturales con el fin de exportarlos sin mayor procesamiento, pudiendo ser minerales, gas, petróleo, materias primas agrarias, forestales y pesqueras.

3 Entendiendo por desarrollo el crecimiento económico sostenido, la equidad social y la estabilidad política. 
de exportaciones (Cepal, 2012). Destacan el cobre en el caso de Chile y Perú, y el petróleo en el de Colombia y Ecuador. El cobre en Chile representaba el 58\% del valor total exportado al mundo en el año 2010 (el año 2000 equivalía al 40\%); mientras que en Perú, representaba el 25\% del valor total exportado (el 2000 era un 14\%). ${ }^{4}$ El petróleo en Colombia representaba el $41 \%$ del valor total exportado (el 2000 era 36\%) y en Ecuador, el 55\% de su valor total exportado (en 2000 era 52\%).

En el caso de Colombia, a diferencia de Chile y Perú, el incremento de la producción extractiva de la mano del auge mundial de las materias primas solo se produjo en el último tiempo, después de que el país ya contaba con una economía relativamente diversificada. Al final del ańo 2000, los hidrocarburos solo representaron poco más del $5 \%$ del PIB nacional; y la minería, aproximadamente un $2 \%$.

Independientemente del color político de los gobiernos de los países aquí analizados, todos han mantenido en los últimos ańos al sector extractivista como pilar preponderante de la economía y del modelo de desarrollo. Aun los progresistas, atraídos por la promesa de contar con ingentes recursos que podrían redistribuir en beneficio del bienestar general de la población, reafirman la extracción de los recursos naturales (Bebbington, 2009; Gudynas, 2009).

Unido a lo anterior, los países seńalados llevaron a cabo reformas estructurales en el marco del Consenso de Washington. Es así como la legislación nacional y el reparto de responsabilidades han transferido mucho poder y liderazgo a las empresas privadas, dejando al sector público un rol más bien facilitador y regulador (Bebbington, 2011b). El caso de Perú grafica lo que ha ocurrido en torno a la minería: desde inicios de los años noventa, el recurso tierra ha sido redistribuido, reclasificado y parcelado para facilitar la transferencia de los derechos mineros a las operaciones mineras de gran escala. Las operaciones mineras trasnacionales están acelerando la transformación de las leyes que rigen la tenencia de tierras, desde el manejo comunal o la negociación informal hacia la propiedad privada; están conduciendo a una revalorización de los recursos y prioridades del uso de tierras, y afectando la distribución vertical de los patrones de uso (Bury, 2011b).

Una de las consecuencias de las reformas neoliberales aplicadas en América Latina es que las actividades minero-extractivas se han convertido en el sector clave del crecimiento económico, de los ingresos liderados por las exportaciones y de la inversión extranjera directa (IED) (Bury, 2011a), volviéndose a las economías rentistadependientes. En el caso de Chile, donde la minería representa el 13,4\% del PIB, en el período 1998-2011 el 30\% del total de la IED se dirigió a dicho sector; y en el período 2002 al 2010, el 84\% de la inversión privada en minería de cobre y oro provino de compañías extranjeras (Calfucura, Martínez, Sanborn \& Dammert, 2013).

No obstante lo anterior, en países como Ecuador se ha desplegado un proceso de nacionalización de recursos naturales y de cambio de rol del Estado, con una mayor presencia y un papel más activo sobre los recursos y los beneficios que su extracción escala mundial creció en 90\%, mientras que en América Latina lo hizo en 400\% y en Perú, un 2000\% (Bebbington, 2011b). De los diez países del mundo que han recibido mayor flujo de inversión entre 1990-2001, cuatro son de América Latina, destacando Chile y Perú (Bebbington, 2011b). 
produce (Acosta, 2011), a objeto de financiar programas sociales, lo que no ha estado exento de complejidades.

\section{Recursos naturales, comunidades y el deseado desarrollo:} ¿con quiénes y para quiénes?

Los recursos naturales ocupan un espacio físico, pero no se trata de tierras "vacías", sino de lugares habitados y configurados como espacios sociales con significados culturales y una historia (Bebbington, 2011a). Las poblaciones que los habitan, y que estructuran su vida en su entorno de la mano de algunos de sus recursos, generalmente agua, son comunidades que la mayoría de las veces no participan de las decisiones relativas a las inversiones y actividades económicas que recaen en el territorio. De hecho, no suelen ser consideradas siquiera a la hora de decidir si sus localidades deberían buscar el "desarrollo" y cómo debiera darse dicho proceso.

En Perú, $72 \%$ del territorio amazónico está cubierto por bloques de hidrocarburos, entre concesiones vigentes y propuestas (Finer \& Orta-Martínez, 2010, citado en Bebbington, 2011b). En Ecuador, cerca de las dos terceras partes de la Amazonía han sido zonificadas para la expansión hidrocarburífera. En ambos países, más de la mitad de las comunidades campesinas se encuentra afectada por las concesiones mineras (De Echave, 2009, ${ }^{6}$ citado en Bebbington, 2011b). Por su parte, en Colombia, a finales de diciembre de 2012, habían sido suscritos 9.400 títulos mineros, cubriendo 5,6 millones de hectáreas. De estos, 3.760 están en explotación y abarcan cerca de 2,1 millones de hectáreas, un área cercana al 1,8\% del territorio nacional. Parte de esos títulos ha sido concedida en zonas de protección ambiental (humedales, áreas de reserva forestal, parque nacionales y regionales, páramos), zonas de nacimientos de agua y de recargas de acuíferos (Velázquez, 2013).

Las actividades extractivas no suelen ser intensivas en empleo ni grandes dinamizadoras locales en cuanto a consumo de insumos para la actividad (Bebbington, 2012); de hecho, el discurso en torno a los desarrollos mineros ya no se centra en la generación de empleo, sino en políticas de responsabilidad corporativa socioambiental y mecanismos de compensación (Damonte, 2011). En Perú, en 2011 las exportaciones mineras representaron el 59\% de las exportaciones totales del país, pero solo alrededor del $1 \%$ de la población se empleaba directamente en la minería. Por otra parte, si bien en su mayoría los insumos utilizados -incluyendo bienes y servicios- fueron de origen nacional, solo el $20 \%$ de ellos corresponde a las regiones con producción minera, proviniendo la gran parte de ellos de la ciudad capital, Lima (Calfucura et al., 2013).

Cfr. M. Finer \& M. Orta-Martínez (2010). A second hydrocarbon boom threatens the Peruvian Amazon: trends, projections, and policy implications. Environmental Research Letters, 5(1). https://doi.org/10.1088/1748-9326/5/1/014012 [N. de E.].

6 Cfr. J. de Echave (2009). Minería y conflictos sociales en el Perú. En J. de Echave, R. Hoetmer \& M. Palacios Panéz (coords.), Minería y territorio en el Perú. Conflictos, resistencias y propuestas en tiempos de globalización (pp. 105-129). Lima: Programa Democracia y Transformación Global. http://www.bibliotecavirtual.info/wp-content/uploads/2011/05/mineria_y_territorio_en_el_ Peru.pdf [N. de E.]. 
La explotación minera a gran escala afecta las estrategias de subsistencia de las comunidades (Bury, 2011b), trastoca, cambia y remueve las bases sobre las cuales se conforman los territorios en espacios rurales: el medio natural y sus recursos, la estructura institucional -acceso y uso de los recursos-y las relaciones sociales, más aún donde tiende a conformar enclaves (Moguillansky, 2013).

La explotación de recursos naturales bajo el modelo extractivista ha significado impactos negativos para las comunidades que habitan en las áreas explotadas. En la minería, son usuales los casos en los que la actividad ha contaminado la tierra y agua de las comunidades campesinas e indígenas, violando sus derechos, a la vez que ha degradado ecosistemas, afectado la salud humana y aumentado su vulnerabilidad (Bebbington, 2009). Y junto con ello, ha perturbado los fundamentos agrarios de los modos comunales de producción y de las economías domésticas. La compra de tierras para fines mineros ha implicado la pérdida de sectores productivos o zonas de producción, restringiendo el acceso de las familias campesinas a ciertos recursos y mermando su actividad económica agropecuaria. También ha significado la monetización de las relaciones en las comunidades y la entrega de grandes sumas de dinero a las familias, las cuales, carentes de las capacidades necesarias para administrarlas, han visto desaparecer su capital en corto tiempo, quedando sin tierra y sin dinero. Por otro lado, ha producido divisiones al interior de las comunidades por obtener los beneficios y compensaciones ofrecidas por las empresas mineras. Finalmente, ha contribuido a la deslegitimación de las autoridades, acusadas de corrupción al participar en constantes negociaciones con los ejecutivos de las compañías (Damonte, 2011).

\section{Conflictos socioterritoriales: tensiones y territorialidades}

En los últimos años, la geografía política latinoamericana aparece signada, de un lado, por disputas sociopolíticas territorialmente localizadas y asociadas a los bienes naturales, los cuales son reclamados como propios por las comunidades; y de otro, por la generación de amplias externalidades negativas que ponen en cuestión tanto las ideas de responsabilidad social empresarial del mundo privado, como el poder de fiscalización y garantía que puede otorgar el Estado.

El territorio no es solo el espacio en el que tiene lugar el conflicto; es su fundamento, la definición de un tipo de territorialidad que incluye la utilización de sus recursos y la creación o resignificación de las relaciones sociales. La definición de lo que es el territorio se convierte en el locus del conflicto (Svampa, 2008b). Esto, pues es en el campo social y material de los territorios donde se construyen las identidades y los proyectos vitales de los actores, así como las estrategias de inversión de las empresas y las políticas públicas gubernamentales.

Es a partir de los procesos de territorialización que se disputa la definición de un determinado tipo de territorialidad (Fernandes, 2005); y es en ese escenario que los movimientos socioterritoriales cobran protagonismo como acción visible y organizada del conflicto. Los movimientos campesinos, los indígenas, las empresas, los sindicatos y los Estados crean relaciones sociales con miras al logro de sus intereses y, de ese modo, producen sus territorios. En dicho proceso entran en tensión distintas territorialidades, siendo un ejemplo paradigmático la minería metalífera 
a gran escala, que se presenta como una visión de territorialidad excluyente de las otras, existentes o potenciales (Porto-Gonçalves, 2009).

Un conflicto socioterritorial supone desacuerdos entre actores con intereses y prioridades diferentes sobre un determinado territorio. El objeto de disputa es el territorio, su definición, uso y significado, elementos generalmente asociados a la estructura de propiedad, al uso y manejo de los recursos naturales y al aprovechamiento de las oportunidades de riqueza o bienestar asociadas. Gran parte de las veces la disputa adopta la forma de conflicto socioambiental, definido como una situación surgida a partir de un impacto ambiental producido por una determinada acción, en torno a la cual se enfrentan tres tipos de actores: empresas que usan recursos ambientales y generan un impacto; la comunidad (o grupos de esta) organizada en torno a la defensa de sus intereses en relación con dicho impacto; y agencias públicas con responsabilidades de mediación en el conflicto (Sabatini, 1994). Además, estos conflictos tienen la particularidad de la multiescalaridad: están anclados a un territorio, pero su alcance lo supera, pues involucra tratados de libre comercio, multinacionales, agencias y ONG (Delamaza, Maillet \& Martínez, 2017).

Considerando los conflictos socioambientales en torno a la actividad extractiva, en América Latina al ańo 2018 se contabilizaron 800 de ellos (Atlas Global de Justicia Ambiental, 2018); y en relación con la minería, llegaron a 254 (ocmaL, 2018). En las últimas tres décadas, los cuatro países acumulan más de 100 conflictos (tabla 1), con diversa duración y radicalidad.

\begin{tabular}{|c|c|c|c|c|}
\hline PAís & Ecuador & Colombia & Perú & Chile \\
\hline TотAL & 8 & 16 & 39 & 43 \\
\hline
\end{tabular}

TABLA I | Conflictos socioambientales mineros en el período 1983-2018

FUENTE: ELABORACIÓN PROPIA CON BASE EN DATOS DEL OBSERVATORIO DE CONFLICTOS MINEROS DE AMÉRICA LATINA (OCMAL) / OBSERVATORIO LATINOAMERICANO DE CONFLICTOS AMBIENTALES (OLCA)

En Ecuador, la lucha contra la megaminería a cielo abierto se inició en 1997, en Cotacachi, constituido en el primer "cantón ecológico" por ordenanza municipal. De ahí en adelante se ha dado con especial intensidad un debate respecto al modelo extractivista-exportador, conflicto reflejado en la división del gabinete del gobierno entre "extractivistas" y "ecologistas" (Ramírez \& Minteguiaga, 2007) y en propuestas presidenciales sin precedentes, como la concepción integral del desarrollo plasmada en el Plan Nacional de Desarrollo 2007-2010 y la de no-explotación del petróleo del parque nacional Yasuni a cambio de una compensación de la comunidad internacional. Todo ello quedó en duda luego de que el gobierno concediera a fines de 2007 la licencia a Petrobras para explotación en un sector de Yasuni, y de que en 2009 la nueva ley minera avalara el modelo, desconociendo el derecho a la oposición y consulta de las poblaciones afectadas por la extracción de los recursos naturales (Svampa, 2008b).

En Perú, la mayoría de conflictos socioambientales fueron producidos por la minería, hidrocarburos, la actividad energética y la forestal (sitio web del Observatorio 
Latinoamericano de Conflictos Ambientales [OLCA], 2013). Destacan los casos de El Espinar (explotación minera que redundó en una mesa de diálogo para la resolución del conflicto); Bagua (paradigmático por las protestas en que participaron miles de indígenas reclamando su reconocimiento como pueblos amazónicos y su rechazo a la usurpación de sus recursos naturales y territoriales; véase Pajuelo, 2009); así como la serie de conflictos en Ancash (32 casos) y Puno (22).

En 1999 surgió la Coordinadora Nacional de las Comunidades del Perú Afectadas por la Minería (Conacami), que articula comunidades y organizaciones de nueve regiones del país. En 2002, en Tambo Grande se realizó la primera consulta popular para la definición del carácter del territorio, siendo el primer plebiscito en esta materia en América Latina. En el año 2007 se realizaron dos consultas más (Piura y Cajamarca) (Svampa, 2008b).

En Colombia, en varias zonas del país (La Colosa, en Tolima; Santurbán, en Santander; Marmato, en Caldas, entre otras), se han movilizado diferentes sectores de la población en contra de la gran minería por sus impactos ambientales (agua, reservas forestales, contaminación de ríos).

Finalmente, en Chile, en las últimas décadas se han venido sucediendo conflictos ambientales asociados a proyectos mineros (Pascua Lama, Pelambres, Isla Riesco), hidroeléctricos o termoeléctricos (Ralco, Hidroaysén, Central Castilla), forestales (Celco, Mehuín) y agroindustriales (Freirina; transgénicos y uso de pesticidas). Las comunidades se han volcado en defensa de sus espacios locales, que se ven seriamente afectados por la intervención de empresas privadas.

Los conflictos y la presión de la crítica pueden ayudar a reformar las políticas estatales y empujar al cambio institucional progresivo (Bebbington, 2012). La protesta y los movimientos socioterritoriales han impulsado cambios que probablemente no hubiesen tenido lugar en su ausencia, tales como la voluntad de la Defensoría del Pueblo, en el Perú, para desempeñar un papel más importante en la gestión de los conflictos sociales o el aumento de la distribución de regalías a nivel regional, así como las nuevas reglas en Ecuador que prohíben la extracción de recursos en ciertas zonas (Kirsch, 2012).

Las preguntas que se busca responder son: ¿qué instrumentos institucionales utilizan los países frente a la actividad extractiva y los conflictos derivados de ella? Y ¿qué nudos críticos y desafíos existen para avanzar hacia un desarrollo inclusivo con equidad territorial en dicho marco?

\section{Métodos y fuentes de información}

La metodología utilizada es la de estudio de casos (Coller, 2005), considerando cuatro países de la región andina, con diverso número de conflictos en torno a la minería: Ecuador, Colombia, Perú y Chile.

A partir de una exhaustiva revisión documental (legislación, estudios, artículos), se identificaron los instrumentos públicos vigentes en los países en torno a la actividad extractiva-minera y se los analizó en su alcance y efectos. Los instrumentos analizados se asocian a la recaudación y distribución de rentas, el ordenamiento territorial, la consulta previa y las leyes ambientales. La tabla 2 describe los instrumentos según país. 


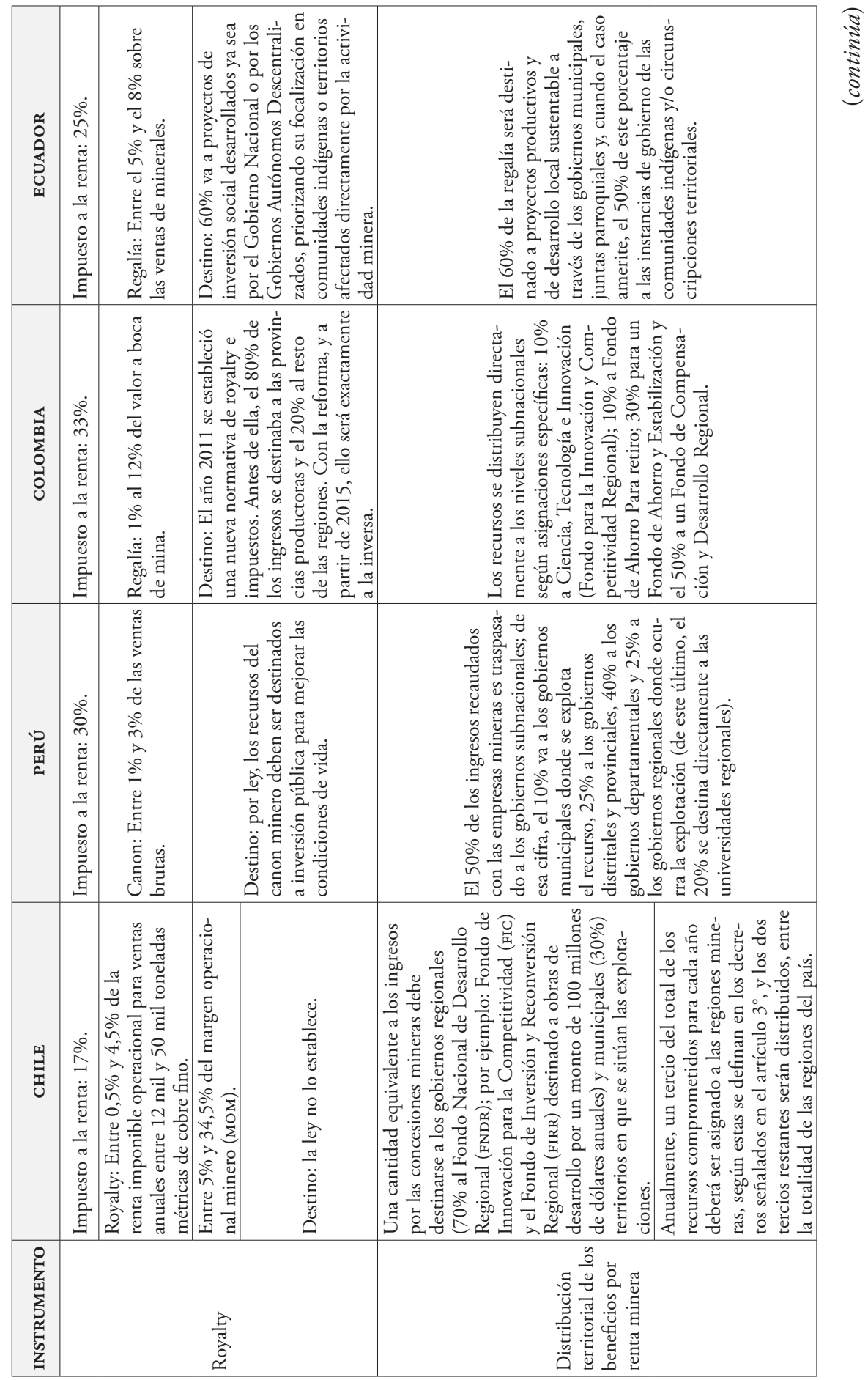




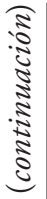

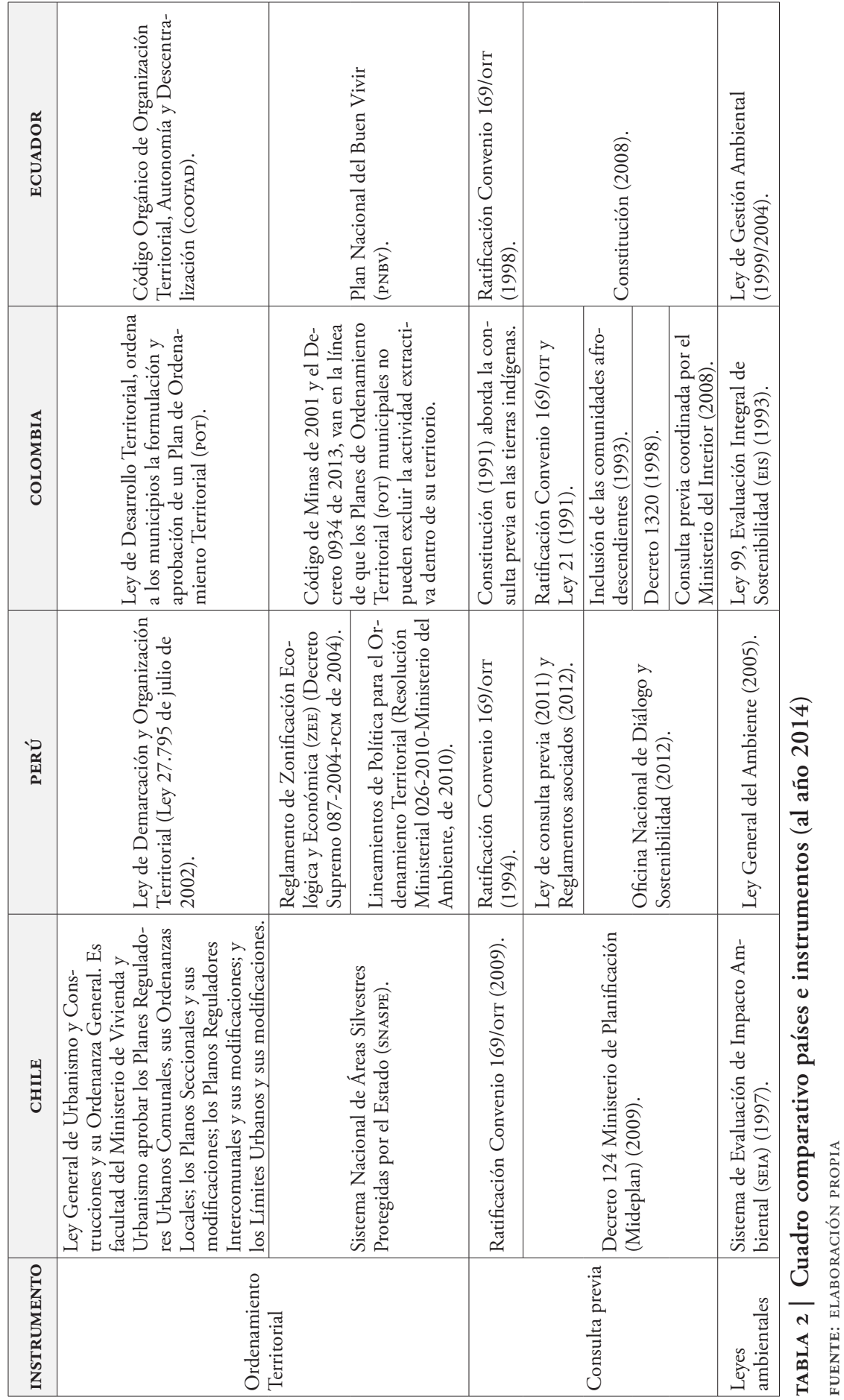




\section{Análisis de instrumentos públicos en torno a la actividad minera y sus conflictos asociados}

El análisis de la respuesta político-institucional en la forma de instrumentos en torno a la actividad minera y los conflictos socioterritoriales derivados de ella, permite comprender de qué modo los Estados entienden dicha actividad económica y sus consecuencias, a la vez que hace posible situarlas en relación con el concepto de territorio y avanzar hacia una reflexión sobre sus posibilidades de eficacia. Más extracción de renta, ordenamiento territorial, procesos de consulta y diálogo son piezas de un rompecabezas que debe ser articulado y mejorado.

\section{Disponibilidad de recursos nacionales y distribución del bienestar:}

la aplicación de royalty

La actividad extractiva, en mayor o menor medida, ha significado la mayor disponibilidad de recursos para los Estados, los cuales han apostado a legitimar esa estrategia de desarrollo mediante la inyección de presupuesto a políticas sociales.

Una manera de escapar de la "maldición de los recursos naturales" es trasladar los beneficios de la economía extractiva a inversiones en sectores de mayor valor agregado. Ya a fines del siglo xix, en Chile el presidente Balmaceda intentó hacerlo. Su plan consistía en captar los excedentes del salitre e invertirlos en capital físico y humano para crear capacidades productivas que estuvieran disponibles cuando el recurso natural ya no otorgara beneficios. Balmaceda estableció un impuesto a las exportaciones de salitre que alcanzaba a un tercio de su valor, y con esos recursos duplicó el número de estudiantes en la educación primaria y secundaria y desarrolló un programa de obras públicas, especialmente ferrocarriles (creó el Ministerio de Obras Públicas e Industria, fomentando también la manufactura). La inversión pública en capital físico se cuadruplicó en términos reales y la de educación se incrementó ocho veces (Palma, 2013). ${ }^{7}$

Más de un siglo después, el planteamiento sigue siendo vigente (André, Berger, Escarfuller, Hayman \& Tummino, 2013). En ese marco, se destacan algunas iniciativas por parte de Chile y Colombia. El Fondo para la Innovación y Competitividad (FIC) en Chile y el 10\% destinado a Investigación y Desarrollo en Colombia apuntan en la dirección de favorecer un motor de desarrollo fuera del campo extractivo. En Perú, si bien el 20\% del canon minero destinado a las universidades regionales es un avance, en la práctica ha dejado de financiar investigación y formación como estaba previsto inicialmente, poniéndose en cuestión su efectividad.

La figura del royalty o canon minero constituye un impuesto adicional al impuesto a la renta, aplicado a los particulares que extraen y se benefician de las riquezas minerales del subsuelo. En los cuatro países existe este instrumento, con distinta antigüedad y con diferencias en sus características.

Lamentablemente, ello quedó truncado al ser contestado por aquellos cuyos intereses iban en otra dirección, estallando la Guerra Civil de 1891 y la disputa por un régimen de poder presidencialista o parlamentarista. 
En Chile, los ingresos fiscales asociados a la actividad minera tienen dos principales caminos. Por un lado, los vinculados a la Corporación Nacional del Cobre de Chile (Codelco) entran directamente al presupuesto nacional, salvo los excedentes de los ingresos proyectados, que son destinados a dos fondos soberanos. Por el otro, los vinculados a las empresas privadas entran al presupuesto público, pero una cantidad equivalente a los ingresos por las concesiones mineras debe destinarse a los gobiernos regionales ( $70 \%$ al Fondo Nacional de Desarrollo Regional [FNDR]) y municipales $(30 \%)$ en los territorios donde se sitúan las explotaciones. Algunos de los destinos de estos recursos han sido el Fondo de Innovación para la Competitividad (FIC) y el Fondo de Inversión y Reconversión Regional (FIRR), destinado a obras de desarrollo (Calfucura et al., 2013).

En Perú, la mayor fuente de ingresos del Estado respecto de la minería es el impuesto a la renta, que representa alrededor del 70\% de su recaudación total de impuestos. Además, las empresas mineras deben pagar derechos y una bonificación anual a sus trabajadores por concepto de utilidades, correspondiente al $8 \%$. También existe el canon minero (que va del 1\% al 3\%), correspondiente a la participación de la que gozan los gobiernos locales y regionales sobre los ingresos y rentas que obtiene el Estado por la explotación de recursos minerales, metálicos y no metálicos. Del $50 \%$ de los ingresos recaudados con las empresas mineras y traspasado a los gobiernos subnacionales, el $25 \%$ va a las regiones y municipios donde se produce la extracción, cifra de la cual el 20\% se destina directamente a las universidades regionales (Instituto Peruano de Economía [IPE], 2011). Por ley, los recursos del canon minero deben ser destinados a inversión pública para mejorar las condiciones de vida; sin embargo, en su mayor parte ha ido a infraestructura (no a salud o educación) y los gobiernos locales usualmente no han tenido capacidad para gestionar y ejecutar dichos recursos (Calfucura et al., 2013).

En Colombia, las regalías mineras pueden ser directas (en beneficio de las entidades territoriales donde se explotan o transportan los minerales) o indirectas (al Fondo Nacional de Regalías). En 2011 se estableció una nueva normativa de royalty e impuestos. Antes de ella, el $80 \%$ de los ingresos se destinaba a las provincias productoras y el 20\% al resto de las regiones; con la reforma, y a partir de 2015, es exactamente lo contrario. Los recursos se distribuyen directamente a los niveles subnacionales según asignaciones específicas: $10 \%$ al Fondo de Ciencia, Tecnología e Innovación (FCTI); 10\% a Fondo de ahorro para retiro; 30\% para un Fondo de ahorro y estabilización y el $50 \%$ a un Fondo de compensación y desarrollo regional.

Finalmente, en Ecuador, las regalías mineras corresponden a un porcentaje que va desde el $5 \%$ al $8 \%$ (el más alto para cobre, oro y plata) sobre las ventas de minerales. Su destino es en un $60 \%$ a proyectos de inversión social desarrollados por el Gobierno Nacional o los Gobiernos Autónomos Descentralizados (GAD), priorizándose su focalización en comunidades indígenas o territorios afectados directamente por la actividad minera. ${ }^{8}$

En todos los países, la mayor disponibilidad de recursos a nivel nacional ha ido de la mano de avances en algunos indicadores sociales, como reducción de la pobreza y 
aumento de capital humano y físico. Sin embargo, los niveles de desigualdad social se han mantenido, o incluso aumentado, y los beneficios generados no siempre han quedado en los territorios explotados.

Un factor clave tiene que ver con las capacidades institucionales de los gobiernos subnacionales, así como con la institucionalidad en materia de transparencia y control de la corrupción. Municipios de territorios tradicionalmente pobres pueden ver incrementados sus ingresos drásticamente sin que existan las planificaciones de gasto adecuadas o los controles necesarios para asegurar su buen uso. El caso de San Marcos, en Perú, es ejemplo de los riesgos y desafíos que comporta la inyección de recursos si no están dadas las condiciones institucionales. El gobierno local de dicho municipio, el más cercano a la mina Antamina, recibió 53 millones de dólares solo el año 2012; sin embargo, la ciudad, con una población de 13.607 habitantes, no tenía agua potable, y dos alcaldes sucesivos han sido acusados de corrupción (André et al., 2013).

\section{Legislación ambiental, planificación y ordenamiento territorial ${ }^{9}$}

Resulta razonable que antes del otorgamiento de concesiones para explorar o extraer gas, petróleo o minerales, ello responda a una definición de las áreas adecuadas para dichos proyectos, y sobre la base de esa definición se determine su viabilidad. En Latinoamérica existen al respecto variados marcos normativos, aunque enfrentan una abierta resistencia por parte de los actores privados (Societas, 2012).

En Perú, destacan la Ley de Demarcación y Organización Territorial (LDOT) (Ley 27.795 de julio de 2002), el Reglamento de Zonificación Ecológica y Económica (ZEE) (Decreto Supremo 087-2004-PCM de 2004), y los Lineamientos de Política para el Ordenamiento Territorial (LPOT) (Resolución Ministerial 026-2010-Ministerio del Ambiente, de 2010). Dentro de la política se plantea como objetivo del ordenamiento territorial "alcanzar el ordenamiento del uso y ocupación del territorio nacional, mediante la Zonificación Ecológica Económica, en un marco de seguridad jurídica y de prevención de conflictos" (Política Nacional Ambiental, Decreto Supremo 012-2009-Ministerio del Ambiente, de 2009. Eje de Política 1, Objetivo 5).

La normativa ambiental ha ido avanzando en forma paralela. En el año 2001 se aprobó el Reglamento de la Ley Orgánica sobre la Conservación y Aprovechamiento Sostenible de la Diversidad Biológica; el año 2004, la Ley Marco del Sistema Nacional de Gestión Ambiental; en 2005, la Ley General del Ambiente; y en 2009, la Política Nacional del Ambiente, luego de la creación del correspondiente ministerio el año 2008.

En Colombia, el Código de Minas de 2001 (Ley 685) regula la exploración y la explotación de los recursos mineros, buscando promover la extracción de un modo sostenible; es decir, utilizando los recursos de manera acorde con la integridad del medioambiente y el desarrollo del país, y estableciendo áreas en las que se reserva el derecho de limitar o excluir la actividad minera. No obstante, la Resolución 0045 del año 2012 define como áreas mineras estratégicas 16 millones de hectáreas,

Parte importante de esta sección se fundamenta en Societas (2012). 
incluidas zonas del Amazonas. Además, tanto el Código de Minas de 2001 como el Decreto 0934 de 2013 van en la línea de que los Planes de Ordenamiento Territorial (РОт) municipales no puedan excluir la actividad extractiva dentro de su territorio. ${ }^{10}$

Ecuador adoptó una nueva Constitución en 2008, en la cual se incluyeron varios conceptos novedosos, como el "buen vivir", el régimen de desarrollo, los derechos de la naturaleza y un nuevo ordenamiento territorial, los que tienen influencia directa sobre las industrias extractivas. Los recursos no renovables son contemplados por la Constitución en una categorización especial, ya que son considerados como un sector estratégico ("aquellos que por su trascendencia y magnitud tienen decisiva influencia económica, social, política o ambiental y deben orientarse al pleno desarrollo de los derechos y al interés social", Art. 313). El Estado se reserva el derecho de administrar, regular, controlar y gestionar los sectores estratégicos, de conformidad con los principios de sostenibilidad ambiental, precaución, prevención y eficiencia. La Constitución determina, asimismo, que los recursos no renovables y la biodiversidad son de propiedad inalienable, imprescriptible e inembargable del Estado (Art. 408).

Las leyes generales y políticas públicas del Ecuador sobre ordenamiento territorial se encuentran contenidas en dos grandes documentos: el Código Orgánico de Organización Territorial, Autonomía y Descentralización (Cootad) y el Plan Nacional de Desarrollo (Plan Nacional del Buen Vivir, pnbv). El Cootad de 2010 regula las disposiciones constitucionales relativas a la organización territorial y la composición político-administrativa del país; reglamenta los Gobiernos Autónomos Descentralizados (GAD), sus funciones y competencias; establece el proceso obligatorio de descentralización y determina disposiciones aplicables al Sistema Nacional de Competencias.

Finalmente, en el caso de Chile, los instrumentos legales disponibles están expresados en la Ley General de Urbanismo y Construcciones y su Ordenanza General, que son de competencia del Ministerio de Vivienda y Urbanismo. Es facultad del Ministerio aprobar los Planes Reguladores Urbanos Comunales y sus Ordenanzas Locales; los Planos Seccionales y sus modificaciones; los Planos Reguladores Intercomunales y sus modificaciones; y los Límites Urbanos y sus modificaciones.

Distintos ministerios tienen competencias también sobre el territorio, tales como el de Medio Ambiente (y su Sistema de Evaluación de Impacto Ambiental); el de Economía, a través de los planes de ordenamiento turístico (pudiendo decretar zonas de interés turístico); o los de Agricultura y de Bienes Nacionales, en lo relativo a definir unidades de manejo en áreas sujetas al Sistema Nacional de Áreas Silvestres Protegidas por el Estado (SNASPE).

Los gobiernos regionales, por su parte, tienen la facultad de formular y gestionar su Estrategia Regional de Desarrollo, con los correspondientes Planes Regionales de Ordenamiento Territorial (РROT) y zonificación del territorio; y las municipalidades deben establecer un Plan de Desarrollo Comunal.

10 "Municipios no podrán decidir sobre minería". Revista Mundo Minero (Colombia), 20 de mayo de 2013. En http://mundominero.com.co/municipios-no-podran-decidir-sobre-mineria/ 


\section{Procesos de consulta previa}

Perú ratificó tempranamente el Convenio oIT/169 (Organización Internacional del Trabajo, sobre Pueblos Indígenas y Tribales en Países Independientes), pero solo en agosto de 2011 se promulgó la Ley de Consulta Previa, cuyos reglamentos se aprobaron en marzo de 2012. Ese año, procesos de diálogo abiertos en torno a la mina Quellaveco (Moquegua) y el proyecto Toquepala arrojaron importantes consecuencias. Junto con el establecimiento de acuerdos, se crearon la Oficina Nacional de Diálogo y Sostenibilidad (onds) y el Servicio de Certificación Ambiental (Senace), representando cambios que han colocado los temas de la consulta, la participación, la planificación territorial y el diálogo en la discusión pública (Bebbington et al., 2013).

En Colombia, la Constitución de 1991 sentó las bases para la consulta previa en las tierras indígenas, de modo que los proyectos no afecten su integridad social, económica o cultural, y ese mismo año adoptó, a través de la Ley 21, el Convenio OIт/169. En 1993 se incluyó a las comunidades afrodescendientes y en 1998 se precisó la ley mediante el Decreto 1320. El año 2008 se le dio institucionalidad al proceso de consulta previa, al entregar la responsabilidad de su coordinación al Ministerio del Interior. La comunidad no tiene poder de veto frente al otorgamiento de licencias, pero puede acudir a los tribunales de justicia (Calfucura et al., 2013).

En Ecuador, tanto la Constitución como la Ley de Participación Ciudadana dan cuenta de los procesos de consulta previa. La Constitución de 2008 es un marco normativo avanzado en la gestión del reconocimiento de los derechos de las comunidades, pueblos y nacionalidades, y del ambiente. Estas leyes se alinean además a acuerdos internacionales suscritos o aprobados por el país, entre los que se encuentran el Convenio oiт/169 ratificado en 1998 y la Declaración de las Naciones Unidas sobre los Derechos de los Pueblos Indígenas, validado en 2007. Sin embargo, han existido importantes reclamos de la representación indígena por la falta de cumplimiento del proceso de Consulta y violación de este Derecho Colectivo previo en los procesos de aprobación tanto de leyes como de proyectos de manejo de recursos naturales, como los petroleros y mineros (Societas, 2012).

El año 2009 Chile ratificó el Convenio oiт/169, institucionalizando el derecho de los pueblos originarios a la consulta previa en aquellos casos en los que proyectos o intervenciones los afecten; sin embargo, el mismo ańo, el Ministerio de Planificación y Cooperación (Mideplan ${ }^{11}$ ) dictó el Decreto 124, que limita dicho proceso de consulta al marco del Sistema de Evaluación de Impacto Ambiental (sEIA) (Calfucura et al., 2013). ${ }^{12}$

Si bien la Consulta Previa constituye una aproximación positiva en la línea de evitar o mitigar la existencia de conflictos, las características que han tomado las normativas correspondientes en Perú, Chile y Colombia, han resultado en incertidumbre. Muchas veces las consultas se realizan después de que los proyectos han

11 Desde 2011, Ministerio de Desarrollo Social; y desde 2019, Ministerio de Desarrollo Social y Familia.

12 Con ello se modificó la naturaleza del convenio, el cual supone que son las propias comunidades indígenas las que deben proponer el procedimiento para ser consultadas, mientras que con la aplicación del decreto es el Estado el que define cuál es el procedimiento y en qué casos corresponde aplicarlo. 
sido diseñados e incluso comenzados a ejecutar, generando impactos socioambientales y la consiguiente respuesta de movilización de las comunidades. Las tensiones entre empresas y comunidades se han trasladado a los tribunales de justicia, generando inseguridad respecto de sus sentencias.

\section{Mecanismos de diálogo}

En cada conflicto entran en juego distintas visiones sobre la naturaleza de los recursos naturales y los beneficios de su explotación, versus los daños ambientales y sociales que genera. Dependerá del curso que tomen los actores involucrados el que se abran espacios de diálogo o de confrontación (y eventual judicialización). ${ }^{13}$ Considerar los intereses, racionalidades, estrategias y discursos de cada uno de los actores y la construcción de campos comunes de interpretación, son requisitos para el manejo de los conflictos (Velásquez, Gaitán, Martínez \& Pérez, 2011). Fundamentales en esta materia son los procesos de diálogo.

La literatura destaca tres enfoques de diálogo para abordar los conflictos socioambientales (Consejo Nacional de Innovación para el Desarrollo [CNID], 2017): i) diálogos colaborativos, que buscan resolver los problemas y sus causas inmediatas a través de acuerdos en el corto plazo (separando necesidades e intereses, abordando estos últimos); ii) diálogos transformativos, que buscan promover cambios sociales (identificando causas estructurales); y iii) diálogos deliberativos, que buscan democratizar las decisiones controversiales a través de mecanismos de deliberación, como foros híbridos con actores sociales y técnicos. El primero supone un relativo equilibrio entre los actores, omitiendo las asimetrías de poder; el segundo opera a largo plazo; y el tercero se ubica a medio camino entre los precedentes.

En la línea de este último estaría el propuesto en Chile por la Alianza Valor Minero (2018), denominado "Diálogo con resultados". Este supone la creación de una Agencia de Diálogo Territorial, encargada de formalizar procesos de diálogo entre Estado, comunidades y privados, y convenir condiciones de sostenibilidad económica, social y ambiental, apoyando el proceso de los proyectos con información, capacidades, facilitación del diálogo, monitoreo de cumplimiento de compromisos y mediación en controversias.

Otro ejemplo cercano al modelo deliberativo es el del proyecto Quellaveco, en la región de Moquegua, Perú. Luego de más de diez años de conflicto, el gobierno regional impulsó la instalación de una mesa de diálogo conformada por representantes de veinte instituciones (organizaciones sociales locales, junta de regantes, representantes municipales, de comunidades y de distintos ministerios), orientada a analizar y elaborar un informe respecto de la viabilidad social, ambiental e hídrica del proyecto minero de la empresa Anglo American. Luego de veinte reuniones en dieciocho meses de trabajo, la citada instancia definió un marco de viabilidad para el proyecto que considera una inversión cercana a los 3.000 millones de dólares. Junto con incorporar algunas recomendaciones relativas al uso de las aguas, se acordó

13 En Chile, a junio de 2013, se identificaron 24 proyectos paralizados, postergados o desistidos, con un monto de inversión asociado superior a los 35.000 millones de dólares, por motivos de incerteza jurídica o judicialización (Sociedad de Fomento Fabril [Sofofa], 2013). 
constituir un Fondo de Responsabilidad Social que deberá operar desde la autorización del proyecto hasta el término de la vida estimada de la mina, con un aporte cercano a los 30 millones de dólares anuales, y la conformación de un Comité de Vigilancia de los compromisos asumidos en la mesa de diálogo, integrado por organizaciones sociales, representantes municipales, del gobierno regional, de la empresa, y la asistencia técnica de un conjunto de ministerios. ${ }^{14}$ Como resultado, el proyecto fue aprobado en julio de 2018 y Anglo American firmó 26 compromisos. Su experiencia, conocida como "modelo Quellaveco", se ha dado a conocer como ejemplo digno de duplicar, aunque está por verse su efectividad una vez que el proyecto avance en su operación.

Responsabilidad Social Empresarial (RSE) y valor compartido: la empresa "total" Desde el propio núcleo del sistema capitalista se reconoce que la acción de las empresas está siendo vista por la ciudadanía como la principal causa de los problemas sociales, ambientales y económicos, reduciéndose de modo considerable sus niveles de legitimidad (Porter \& Kramer, 2011). Porter y Kramer, de la Escuela de Negocios de Harvard, señalan la necesidad de conectar el éxito empresarial con el progreso social, para lo cual proponen la idea de "valor compartido", definido como "las políticas y prácticas operacionales que aumentan la competitividad de una empresa, mientras simultáneamente mejoran las condiciones sociales y económicas de las comunidades en las cuales opera" (p. 3). El planteamiento de los autores es llevar al núcleo de la empresa aquello que estuvo siempre en la periferia de sus preocupaciones y que con el tiempo hizo que los Estados, presionados por la sociedad civil, establecieran regulaciones y normativas destinadas a cautelar ciertas condiciones mínimas.

La idea de valor compartido suena sugerente; sin embargo, pone en el centro la acción empresarial y su necesidad de creación de valor, no ya forzada por el Estado o por la sociedad a cumplir con ciertas regulaciones o aportar económicamente a las comunidades, sino por su propio interés y su necesidad de ganar en competitividad. La empresa se expande y absorbe su entorno, incorporándolo tras su ideal de creación de riqueza, erigiéndose como "institución total” (à la Goffman) en el territorio.

\section{Discusión y reflexiones finales: la gobernanza territorial para un desarrollo inclusivo debe ir más allá de la Responsabilidad Social Empresarial (RSE) y el valor compartido}

En la actualidad, las inversiones de gran escala enfrentan nuevos desafíos. Pese a los esfuerzos en algunos ámbitos, los conflictos no han cesado y los contextos institucionales no han resuelto los problemas y contradicciones entre la industria extractiva y las comunidades.

Es necesario observar la situación de la economía extractiva con otros lentes, comprendiendo que los conflictos socioterritoriales no se resolverán por obra de

14 Véase nota "Anglo American aprueba el inicio del proyecto Quellaveco", en https://peru. angloamerican.com/medios/noticias/2018/2018-07-26?sc_lang=es-es 
una empresa. Por el contrario, como se ha venido describiendo aquí, se debe reconocer la participación de distintos actores que conforman territorialidades con sus intereses e identidades, por lo cual solo los procesos de articulación, diálogo y negociación, en un marco de respeto de derechos, harán posible avanzar en el desarrollo de proyectos que otorguen bienestar social.

A la luz de lo revisado, se propone profundizar en los instrumentos actuales, acompańándolos de procesos de fortalecimiento de los actores territoriales, de sus capacidades de articulación, diálogo y gestión. La dirección de los cambios propuestos va hacia:

- Fomentar y refinar los instrumentos jurídicos de captura de excedentes derivados de la actividad extractiva, para dirigirlos a inversión en otras áreas de desarrollo, las cuales configuren la base para una diversificación productiva y la generación de capacidades humanas y materiales.

- Desarrollar instrumentos de planificación y ordenamiento territorial que supongan la existencia de acuerdos sobre la historia, vocaciones y perspectivas de los territorios, pudiendo arribar a equilibrios relativos entre zonas especialmente valoradas para la conservación y zonas aptas para la actividad extractiva, pero en respeto de las condiciones sociales y medioambientales que reduzcan al mínimo sus impactos negativos.

- Definir y perfeccionar procesos de consulta previa. Se requieren instrumentos vinculantes, cuya oportunidad, y la capacidad institucional para dirigirlos, permitan un alto grado e intensidad de participación de la población.

- Avanzar en mayor descentralización, fortaleciendo a los gobiernos subnacionales. El liderazgo, las capacidades institucionales y su probidad, así como la oportunidad temporal de las acciones, son aspectos clave para abordar los conflictos. La proximidad territorial, el conocimiento de las comunidades y la capacidad de interacción permanente con la ciudadanía hacen de los gobiernos locales y regionales, actores indispensables. Para que puedan ejercer su papel de manera óptima, no solo deben contar con las competencias y prerrogativas que se lo permitan, sino también con las capacidades requeridas y mecanismos para la rendición de cuentas (accountability) que garanticen la adecuada representación de los intereses comunes y el buen destino de los recursos involucrados.

- Fortalecer la capacidad de concertación y articulación de los actores para arribar a arreglos institucionales que permitan el crecimiento económico y el desarrollo inclusivo. La resolución de conflictos pasará por lograr arreglos institucionales multiactorales (comunidades, empresas, Estado) y una institucionalidad estatal que sea garante de los derechos de quienes habitan los territorios intervenidos extractivamente, así como activa en la planificación y conducción de procesos de crecimiento económico más dirigidos hacia la diversificación productiva y la equidad territorial que los hoy vigentes.

El fortalecimiento de las capacidades institucionales del sector público y de la sociedad civil, así como la apertura de espacios de diálogo público-privado, deben desarrollarse de manera simultánea a la instalación de prácticas de recaudación y 
distribución de rentas, ordenamiento territorial y consulta previa. Con todos los actores en la mesa, pero con marcos e instrumentos legales que aseguren una relativa simetría de poder entre ellos -una simetría que permita negociar-, será posible arribar a arreglos institucionales eficaces para el desarrollo territorial sobre la base de consideraciones económicas, sociales, culturales y ambientales.

\section{Referencias bibliográficas}

Acosta, A. (2009). La maldición de la abundancia. Quito: Comité Ecuménico de Proyectos (CEP) / SwissAid / AbyaYala. http://www.rebelion.org/docs/122604.pdf

Acosta, A. (2011). Extractivismo y neoextractivismo: Dos caras de la misma maldición. http:// www.polodemocratico.co/pdf/Alberto\%20Acosta.pdf

André, R., Berger, R., Escarfuller, W., Hayman, M. \& Tummino, A. (2013). The good, the bad and the ugly. Cases, comparisons and conclusions. Americas Quarterly, Especial section (Winter). https://bit.ly/1LWC6LC

Atlas Global de Justicia Ambiental (2018). Recurso interactivo. ғuнem Ecosocial. http://www. fuhem.es/ecosocial/noticias.aspx?v=9582\&n=0, 2018

Bebbington, A. (2009). Nuevas políticas extractivas en América Latina. La paradoja de la riqueza: ¿Cómo traducir rentas extractivas en desarrollo territorial? En Memoria del Seminario Internacional Bolivia Post-Constituyente. Tierra, territorio y autonomías indigenas. La Paz, Bolivia 26, 27 y 28 de octubre 2009: International Land Coalition - Fundación Tierra.

Bebbington, A. (2011a). Elementos para una ecología política de los movimientos sociales y el desarrollo territorial en zonas mineras. En A. Bebbington (ed.), Minería, movimientos sociales y respuestas campesinas. Una ecología politica de transformaciones territoriales (pp. 53-76). Lima: Instituto de Estudios Peruanos (IEP) / Centro Peruano de Estudios Sociales (CEPES).

Bebbington, A. (ed.) (2011b). Minería, movimientos sociales y respuestas campesinas. Una ecología politica de transformaciones territoriales. Lima: Instituto de Estudios Peruanos (IEP) / Centro Peruano de Estudios Sociales (CEPes).

Bebbington, A. (Ed.) (2012). Social conflict, economic development and extractive industry. Evidence from South America. Londres: Routledge. https://doi.org/10.4324/9780203639030

Bebbington, A., Escobal, J., Soloaga, I. \& Tomaselli, A. (2016). Trampas territoriales de pobreza, desigualdad y baja movilidad social. Los casos de Chile, México y Perú. Ciudad de México: Centro de Estudios Espinosa Yglesias.

Bebbington, A., Scurrah, M. \& Chaparro, A. (2013). Minería, conflictividad y la política: ¿Algo cambia? Revista Argumentos, 7(1-5). http://www.revistargumentos.org.pe/ mineria_conflictividad_política.html

Bengoa, J. (ed.). (2007). Territorios rurales. Movimientos sociales y desarrollo territorial rural en América Latina. Santiago de Chile: Rimisp / Catalonia.

Berdegué, J. A.; Bebbington, A. \& Escobal, J. (2015). Conceptualizing spatial diversity in Latin American rural development: structures, institutions, and coalitions. World Development, 73, 1-10. https://doi.org/10.1016/j.worlddev.2014.10.015 
Bury, J. (2011a). Neoliberalismo, minería y cambios rurales en Cajamarca. En A. Bebbington (ed.), Minería, movimientos sociales y respuestas campesinas. Una ecología politica de transformaciones territoriales (pp. 79-110). Lima: Instituto de Estudios Peruanos (IEP) / Centro Peruano de Estudios Sociales (CEPes).

Bury, J. (2011b). Minería, migración y transformaciones en los medios de subsistencia en Cajamarca, Perú. En A. Bebbington (ed.), Minería, movimientos sociales y respuestas campesinas. Una ecología política de transformaciones territoriales (pp. 261-308). Lima: Instituto de Estudios Peruanos (IEP) / Centro Peruano de Estudios Sociales (CEPES).

Calfucura, E., Martínez, A., Sanborn, C. \& Dammert, L. (2013). Natural resource extraction. the good, the bad and the ugly. Americas Quarterly, Especial section. Winter. https:// bit.ly/1LWC6LC

Coller, X. (2005). Estudio de casos. Cuadernos Metodológicos No 30. Madrid: Centro de Investigaciones Sociológicas.

Comisión Económica para América Latina y el Caribe (Cepal) (2012). Panorama de la inserción internacional de América Latina y el Caribe. Crisis duradera en el centro y nuevas oportunidades para las economías en desarrollo. Documento informativo 2011-2012. https://bit.ly/2GKLpoN

Consejo Nacional de Innovación para el Desarrollo (CNID), Chile (2017). Evaluación de los conflictos socio-ambientales de proyectos de gran tamaño con foco en agua y energía para el periodo 1998 al 2015. [Asesoría desarrollada por AGEA]. https://bit.ly/2HGxmxA

Damonte, G. (2011). Minería y política. La recreación de luchas campesinas en dos comunidades andinas. En A. Bebbington (ed.), Minería, movimientos sociales y respuestas campesinas. Una ecología politica de transformaciones territoriales (pp. 147-192). Lima: Instituto de Estudios Peruanos (IEP) / Centro Peruano de Estudios Sociales (CEPes).

De Sousa Santos, B. \& Rodríguez, C. (eds.). (2007). El derecho y la globalización desde abajo. Hacia una legalidad cosmopolita. Barcelona: Anthropos.

Delamaza, G., Maillet, A., \& Martínez, C. (2017). Socio-territorial conflicts in Chile: Configuration and politicization (2005-2014). European Review of Latin American and Caribbean Studies (104) (July-December), 23-46. https://doi.org/10.18352/ erlacs. 10173

Fernandes, B. (2005). Movimentos socioterritoriais e movimentos socioespaciais: Contribuição teórica para uma leitura geográfica dos movimentos sociais. En OSAL, Observatorio Social de América Latina, 6(16). Buenos Aires: Consejo Latinoamericano de Ciencias Sociales (Clacso).

Front Line Defenders (FLD) (2018). Annual Report on Human Rights Defenders at Risk in 2017. Dublín: Front Line, the International Foundation for the Protection of Human Rights Defenders. https://www.frontlinedefenders.org/sites/default/files/annual_report_digi tal.pdf

Front Line Defenders (FLD) (2019). Análisis global de Front Line Defenders 2018. Dublín: Front Line, the International Foundation for the Protection of Human Rights Defenders. https://bit.ly/2Wa4wNI 
Gudynas, E. (2009). Diez tesis urgentes sobre el nuevo extractivismo. Contextos y demandas bajo el progresismo sudamericano actual. En J. Schuldt, A. Acosta, A. Barandiarán, M. Folchi, CEDlA-Bolivia, A. Bebbington, A. Alayza \& E. Gudynas, Extractivismo, politica y sociedad (pp. 187-225). Quito: Centro Andino de Acción Popular (CAAP) / Centro Latino Americano de Ecología Social (ClAES) / Fundación Rosa Luxemburg. https:// bit.ly/2IMAOvY

Instituto Peruano de Economía (IPE) (2011). La tributación minera en el Perú: contribución, carga tributaria y fundamentos conceptuales. Lima: IPE. https://bit.ly/2IMAQnAKarl, T. (1997). The paradox of plenty: Oil booms and petro-states. Berkeley, CA: University of California Press.

King, A. (2011). ¿'Hay una estrategia de empleo en el desarrollo económico local? El reto del trabajo decente. En P. Vergara \& F. Alburquerque (eds.), Desarrollo económico territorial. Respuesta sistémica a los desafíos del empleo (pp. 57-76). Fortaleza, Brasil: Red de Desarrollo Territorial de América Latina y el Caribe (Red DETE-ALC).

Kirsch, S. (2012). Afterword: extractive conflicts compared. En A. Bebbington (ed.), Social conflict, economic development and extractive industry. Evidence from South America (pp. 201-213). Londres: Routledge. https://doi.org/10.4324/9780203639030

Moguillansky, G. (2013, nov. 28). El programa de la Nueva Mayoría y el desarrollo regional: las potencialidades truncas del desarrollo de la gran minería. El Mostrador, https://bit. ly/2GAjLts

Observatorio de Conflictos Mineros de América Latina (ocmal) (2018). https://www.ocmal. org/

Observatorio Latinoamericano de Conflictos Ambientales (OLCA) (2013). http://www.olca.cl/ oca/index.htm

Pajuelo, R. (coord.) (2009). Documento de Trabajo No 158, realizado por el Comité de Seguimiento del Conflicto Social y la Coyuntura Latinoamericana de Perú. Informe de Coyuntura Observatorio Social de América Latina (osaL). Publicación electrónica.

Palma, G. (2013). ¿ Y dónde fueron a parar los excedentes del boom del cobre? CIPER, Opinión, 16 abril. https://bit.ly/2vm6OOJ

Porter, M. \& Kramer, M. (2011, Jan/Feb). Creating shared value: redefining capitalism and the role of the corporation in society. Harvard Business Review, 89(1/2), 62-77. https:// hbs.me/2UVehmS

Porto-Gonçalves, C. (2009). De saberes y de territorios: diversidad y emancipación a partir de la experiencia latino-americana. Polis, 8(22), 121-136. http://dx.doi.org/10.4067/ S0718-65682009000100008

Ramírez, F. \& Minteguiaga, A. (2007). El nuevo tiempo del Estado. La política posneoliberal del correísmo. OSAL (22). Buenos Aires: Consejo Latinoamericano de Ciencias Sociales (Clacso). https://bit.ly/2voj2WV

Rimisp, Centro Latinoamericano para el Desarrollo Rural (2012a; 2014; 2016; 2018). Informe Latinoamericano Pobreza y Desigualdad (Serie Bienal). Santiago de Chile: Rimisp. https://bit.ly/2IWXEQI

Rimisp, Centro Latinoamericano para el Desarrollo Rural (2012b). Territorios rurales en movimiento. Informe final del Programa Dinámicas Territoriales Rurales 2007-2012. Santiago de Chile: Rimisp. https://bit.ly/2UMhbFS 
Sabatini, F. (1994). Espiral histórica de conflictos ambientales: el caso de Chile. En Ambiente y Desarrollo, 10(4), 15-22. https://bit.ly/2L3iWi8

Schejtman, A. \& Berdegué, J. (2004). Rural territorial development. Documento de Trabajo No 4. Programa Dinámicas Territoriales Rurales. Santiago de Chile: Rimisp, Centro Latinoamericano para el Desarrollo Rural.

Seoane, J. (2012). Neoliberalismo y ofensiva extractivista. Actualidad de la acumulación por despojo, desafíos de Nuestra América. Revista Theomai (26). Segundo semestre. https://bit.ly/2V2rzxZ

Serrano, C. (2010). Politicas de desarrollo territorial en Chile. Documento de trabajo. Santiago de Chile: Rimisp, Centro Latinoamericano para el Desarrollo Rural. https://bit. ly/2GLbS5C

Serrano, C. \& Ramírez, E. (2013). Empresas y su vinculación con el territorio, innovar para avanzar. Propuesta para aportar a resolver conflictos entre empresas de gran escala y el territorio. Documento de trabajo. Santiago de Chile: Rimisp, Centro Latinoamericano para el Desarrollo Rural.

Societas. Consultora de Análisis Social (2012). Ordenamiento territorial en América Latina en el contexto del desarrollo de las industrias extractivas. Informe final preparado para Reveneu Watch Institute.

Sociedad de Fomento Fabril (Sofofa) (2013). Observatorio de la Inversión. Informativo $N^{\circ} 8$. Santiago de Chile: Sofofa, Departamento de Estudios.

Svampa, M. (2008a). Cambio de época. Movimientos sociales y poder politico. Buenos Aires: Siglo xxi / Consejo Latinoamericano de Ciencias Sociales (Clacso).

Svampa, M. (2008b) La disputa por el desarrollo: territorio, movimientos de carácter socioambiental y discursos dominantes. En M. Svampa, Cambio de época. Movimientos sociales y poder político. Buenos Aires: Siglo XXI / Consejo Latinoamericano de Ciencias Sociales (Clacso). https://bit.ly/2IL02e4

Thorp, R., Orihuela, J. \& Paredes, M. (2013). Avoiding the resource curse. Americas Quarterly (Winter). https://bit.ly/2UD7aKZ

Valor Minero. (2018). Agencia de diálogo territorial para proyectos de inversión. Propuesta de politica pública. https://bit.ly/2IKGHtc

Velásquez, F. (2013, julio). Industrias extractivas y conflictos en Colombia (РРт). Quito: Fundación FORO. https://bit.ly/2vm7pzX

Velásquez, F., Gaitán, L., Martínez, M. \& Pérez, P. (2011). El sector extractivo en Colombia. Bogotá: Revenue Watch Institut / Evangelischer Entwicklungsdienst (EED) / Foro Nacional por Colombia.

\section{Sitios Web}

Atlas Global de Justicia Ambiental: https://ejatlas.org/

Observatorio Latinoamericano de Conflictos Ambientales (OLCA): www.olca.cl

Observatorio de Conflictos Mineros de América Latina (OCMAL): www.ocmal.org 